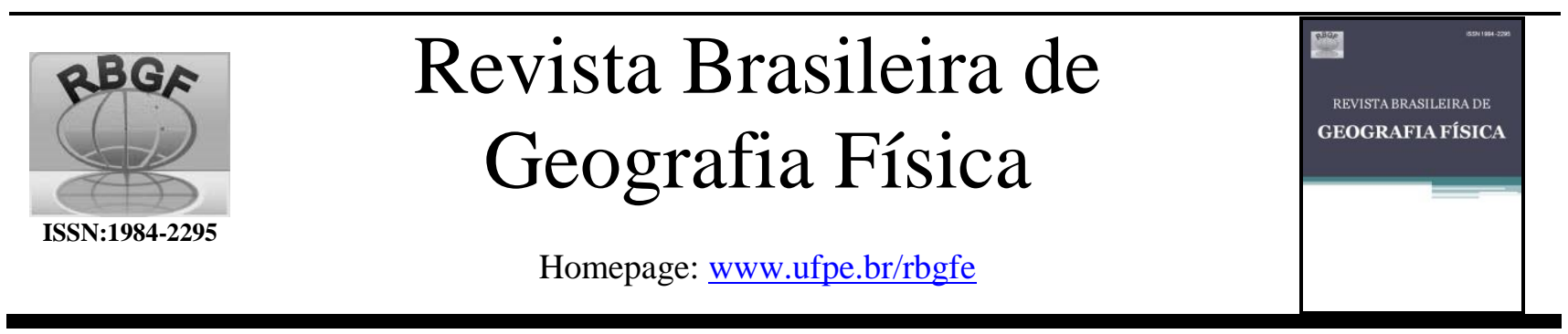

\title{
Qualidade Ambiental de Áreas de Pastagem por Meio de Atributos Visuais ${ }^{1}$
}

\author{
Kelly Rejane de Souza²; Rogério Melloni ${ }^{3}$; Gustavo Magno dos Reis Ferreira ${ }^{4}$
}

${ }^{2}$ Bióloga, mestre em Meio Ambiente e Recursos Hídricos. Instituto de Recursos Naturais (IRN), Universidade Federal de Itajubá (Unifei), Avenida BPS, 1303. CEP 37.500-903. Itajubá, MG. Autor correspondente: E-mail: kelfloresta@hotmail.com. ${ }^{3}$ Engenheiro agrônomo. Prof. Dr. IRN, Unifei. E-mail: rogerio.melloni@ gmail.com. ${ }^{4}$ Biólogo, doutor em Microbiologia Agrícola, Universidade Federal de Lavras, Departamento de Biologia (DBI). Av. Doutor Sylvio Menicucci, 1001 - Lavras, MG, 37200-000. E-mail: gustavo_mrf@yahoo.com.br.

Artigo recebido em 21/04/2018 e aceito em 25/07/2018

R E S U M O

Atividades agrícolas apresentam diversos impactos ao meio ambiente, alterando as características físicas, químicas e biológicas do solo. Um dos principais impactos causados pela atividade pastoril é a compactação do solo, levando a processos de erosão e comprometimento dos atributos físicos, químicos e biológicos do solo. No entanto, há diversas técnicas que permitem o monitoramento da qualidade ambiental dessas áreas com o objetivo de avaliar níveis de degradação, bem como, os processos de recuperação mais adequados. Devido à necessidade de se utilizar métodos sensíveis, eficientes e de baixo custo, os indicadores visuais da qualidade do solo podem ser utilizados visando o manejo dessas áreas. Assim, o objetivo do presente trabalho foi avaliar a qualidade ambiental de treze áreas de pastagens, sob diferentes condições de manejo, por meio de indicadores visuais relacionados a solo, plantas e fauna, pela metodologia de "Referência para Avaliação da Qualidade do Solo" (RAQS). Os resultados mostram que a avaliação de áreas de pastagem, por meio de atributos visuais relacionados a solo, plantas e fauna, possibilita, com baixo custo e eficiência, acesso a sua qualidade ambiental. No entanto, essa qualidade é fortemente sensível e influenciada pelos atributos relacionados à vegetação e ao solo, exceto fauna silvestre (vertebrados), cor e odor do solo e compactação, que não foram considerados bons indicadores da qualidade dos solos de pastagem.

Palavras-chave: análise visual, impactos ambientais, qualidade do solo.

\section{Environmental Quality of Pasture Areas by Visual Attributes}

\section{A B S T R A C T}

Agricultural activities have several impacts on the environment, altering the physical, chemical and biological characteristics of the soil. The main impacts caused by pasture activity are the soil compaction, leading to erosion processes and compromising its physical, chemical and biological attributes. However, there are several techniques that allow the monitoring of the environmental quality of these areas with the objective of evaluating levels of degradation, as well as the most adequate recovery processes. Due to the need to use sensitive, efficient and low-cost methods, visual indicators of soil quality can be used to manage these areas. The objective of the present study was to evaluate the environmental quality of thirteen pasture areas, under different management conditions, through visual indicators related to soil, plants and fauna, using the "Reference for Soil Quality Assessment" (RAQS). The results show that the evaluation of pasture areas, through visual attributes related to soil, plants and fauna, allows, with low cost and efficiency, access to its environmental quality. However, this quality is strongly sensitive and influenced by attributes related to plants and soil, except wild fauna

\footnotetext{
${ }^{1}$ Artigo extraído da dissertação de Mestrado da primeira autora.
} 
(vertebrates), soil color and odor, and compaction, which were not considered good indicators of the soil quality under pastures.

Keywords: visual analysis, environmental impacts, soil quality.

\section{Introdução}

Áreas de pastagens podem comprometer a qualidade do solo (Cavagnaro et al., 2016), devido a processos de erosão e desertificação (Kosmas et al., 2016). Cherubin et al. (2015) demonstraram que a expansão agropecuária pode reduzir significativamente a disponibilidade de nutrientes do solo, além de promover a sua acidificação. Perdas de $\mathrm{C}$ e $\mathrm{N}$, aumentos na quantidade de $\mathrm{CO}_{2}$ liberado e lixiviação do $\mathrm{N}$ também têm sido evidenciadas (Mudge et al., 2016), além de graves problemas de compactação do solo e erosão hídrica em áreas de pastagens em função do pisoteio dos animais (Pulido et al., 2016).

Estudos de qualidade do solo devem ser utilizados por agricultores, extensionistas e cientistas para melhor gestão das áreas e das culturas agrícolas (Askari e Holden, 2015). Estudos relacionados aos atributos físicos do solo são fundamentais para a manutenção da sustentabilidade ecológica desse sistema, pois caracterizam maior controle da movimentação das águas, penetração das raízes, crescimento das plantas e disponibilidade de nutrientes (Naderi-Boldaji e Keller, 2016). Assim, um solo de boa qualidade envolve aspectos físicos, químicos e biológicos (Cherubin et al., 2015; Crittenden et al., 2015), sendo que a degradação desses atributos pode influenciar, tanto a manutenção do funcionamento biológico do solo frente a alterações em seu uso (Fusaro et al., 2018; Raiesi e Kabiri, 2016), como a sustentabilidade econômica e agrícola (Bunemann et al., 2018).

A avaliação de alguns dos atributos de solos, usualmente utilizados para determinação da sua qualidade, depende do emprego de técnicas de difícil execução, de infraestrutura laboratorial sofisticada ou de alto custo. Dessa forma, alguns autores sugerem a utilização de atributos alternativos para definir o IQS, como a utilização de atributos visuais (Nicholls et al., 2004; Souza et al., 2016). Os métodos para avaliar a qualidade visual do solo, entretanto, são semi-quantitivos e aplicados, principalmente, aos atributos físicos da parte superficial do solo, considerados, por alguns autores, como mais importantes nessa avaliação (Guimarães et al., 2013), e complementados por outros diferentes métodos, selecionados de acordo com as condições de cada local.

A avaliação visual pode ser considerada confiável não somente para determinar a qualidade do solo, mas igualmente para determinar a dinâmica de recuperação de áreas degradadas para preservação do solo e manutenção da sustentabilidade (Moncada et al., 2014). No sul de Minas Gerais, as pastagens abrangem grandes áreas (Oliveira et al., 2015) e tendem a apresentar baixo índice de qualidade do solo devido à intensidade do pastejo (Paz-Kagan et al., 2016). Asssim, o objetivo do presente trabalho foi avaliar a qualidade de solos sob pastagem, em diferentes níveis de degradação, por meio de indicadores visuais de forma a contribuir para a definição de estratégias de manejo condizentes com sua sustentabilidade ambiental.

\section{Material e métodos}

O trabalho foi conduzido no município de Itajubá, sul do estado de Minas Gerais, Brasil. Pela classificação climática, a área apresenta, em sua maioria, o clima Cwa (Köppen classification). Os tipos de solo mais recorrentes são, Argissolos (56\%), Cambissolos Háplicos (26\%), Gleissolos (7\%) e Latossolos (6\%), inseridos no bioma Mata Atlântica e vegetação primária definida como Floresta Estacional Semidecidual.

Treze áreas de pastagens foram selecionadas para estudo, localizando-se na microbacia do Ribeirão José Pereira (Itajubá/MG), as quais representam $49,8 \%$ da sua área total, cujas características e localização estão relacionadas na Tabela 1.

Tabela 1. Descrição dos ambientes submetidos à avaliação visual da qualidade do solo 
Revista Brasileira de Geografia Física v.11, n.05 (2018) 1776-1785.

\begin{tabular}{|c|c|c|c|c|}
\hline Ambiente & Características & $\begin{array}{l}\text { Coordenadas } \\
(23 \mathrm{~K}-\mathrm{UTM})\end{array}$ & $\begin{array}{c}\text { Declividade (\%) } \\
\text { e } \\
\text { Relevo } \\
\end{array}$ & Tipo de Solo \\
\hline PB1 & $\begin{array}{l}\text { Pasto visualmente bom*, em área } \\
\text { entre um pasto em sucessão a } \\
\text { floresta e a zona urbana }\end{array}$ & $\begin{array}{c}455864 \\
7522207\end{array}$ & $\begin{array}{l}0-3 \% \\
\text { Plano }\end{array}$ & $\begin{array}{l}\text { Gleissolo Háplico } \\
\text { Distrófico }\end{array}$ \\
\hline CAP1 & $\begin{array}{l}\text { Capoeira, pasto em estágio } \\
\text { intermediário de sucessão, entre } \\
\text { pasto, floresta e zona urbana }\end{array}$ & $\begin{array}{c}455966 \\
7522424\end{array}$ & $\begin{array}{l}8-20 \% \\
\text { Ondulado }\end{array}$ & $\begin{array}{l}\text { Argissolo Vermelho } \\
\text { Distrófico }\end{array}$ \\
\hline PN1 & $\begin{array}{l}\text { Pasto com início de degradação, } \\
\text { em zona rural }\end{array}$ & $\begin{array}{c}456882 \\
7520765\end{array}$ & $\begin{array}{l}8-20 \% \\
\text { Ondulado }\end{array}$ & $\begin{array}{l}\text { Gleissolo Háplico } \\
\text { Distrófico }\end{array}$ \\
\hline PN2 & Pasto degradado, em zona rural & $\begin{array}{c}457089 \\
7520527\end{array}$ & $\begin{array}{l}8-20 \% \\
\text { Ondulado }\end{array}$ & $\begin{array}{l}\text { Gleissolo Háplico } \\
\text { Distrófico }\end{array}$ \\
\hline PB2 & $\begin{array}{l}\text { Pasto visualmente bom, em zona } \\
\text { rural }\end{array}$ & $\begin{array}{c}457810 \\
7519879\end{array}$ & $\begin{array}{c}20-45 \% \\
\text { Forte Ondulado }\end{array}$ & $\begin{array}{l}\text { Argissolo Vermelho } \\
\text { Distrófico }\end{array}$ \\
\hline PB3 & $\begin{array}{l}\text { Pasto visualmente bom, em zona } \\
\text { rural próximo à Reserva } \\
\text { Biológica Serra dos Toledos }\end{array}$ & $\begin{array}{c}461120 \\
7519866\end{array}$ & $\begin{array}{c}20-45 \% \\
\text { Forte Ondulado }\end{array}$ & $\begin{array}{l}\text { Cambissolo Háplico } \\
\text { Distrófico }\end{array}$ \\
\hline PB4 & $\begin{array}{l}\text { Pasto visualmente bom, próximo } \\
\text { a um pasto em sucessão, floresta } \\
\text { e área urbana }\end{array}$ & $\begin{array}{c}455895 \\
7522608\end{array}$ & $\begin{array}{l}8-20 \% \\
\text { Ondulado }\end{array}$ & $\begin{array}{l}\text { Argissolo Vermelho } \\
\text { Distrófico }\end{array}$ \\
\hline CAP2 & $\begin{array}{l}\text { Capoeira, pasto em estágio } \\
\text { intermediário de sucessão, entre } \\
\text { pasto, floresta e estrada na zona } \\
\text { rural }\end{array}$ & $\begin{array}{c}457366 \\
7521670\end{array}$ & $\begin{array}{c}20-45 \% \\
\text { Forte Ondulado }\end{array}$ & $\begin{array}{l}\text { Gleissolo Háplico } \\
\text { Distrófico }\end{array}$ \\
\hline PB5 & $\begin{array}{l}\text { Pasto visualmente bom, próximo } \\
\text { à estrada na zona rural }\end{array}$ & $\begin{array}{c}457018 \\
7521541\end{array}$ & $\begin{array}{c}20-45 \% \\
\text { Forte Ondulado }\end{array}$ & $\begin{array}{l}\text { Argissolo Vermelho } \\
\text { Distrófico }\end{array}$ \\
\hline PB6 & $\begin{array}{l}\text { Pasto visualmente bom, próximo } \\
\text { à estrada na zona rural }\end{array}$ & $\begin{array}{c}453770 \\
7522491\end{array}$ & $\begin{array}{c}8-20 \% \\
\text { Ondulado }\end{array}$ & $\begin{array}{l}\text { Argissolo Vermelho } \\
\text { Distrófico }\end{array}$ \\
\hline CAP3 & $\begin{array}{l}\text { Capoeira, pasto em estágio } \\
\text { intermediário de sucessão, entre } \\
\text { pasto, floresta e estrada na zona } \\
\text { rural }\end{array}$ & $\begin{array}{c}454005 \\
7522426\end{array}$ & $\begin{array}{c}3-8 \% \\
\text { Suave Ondulado }\end{array}$ & $\begin{array}{l}\text { Argissolo Vermelho } \\
\text { Distrófico }\end{array}$ \\
\hline CAP4 & $\begin{array}{l}\text { Capoeira, pasto em estágio } \\
\text { intermediário de sucessão, entre } \\
\text { pasto, floresta e estrada na zona } \\
\text { rural }\end{array}$ & $\begin{array}{c}454904 \\
7520902\end{array}$ & $\begin{array}{c}20-45 \% \\
\text { Forte Ondulado }\end{array}$ & $\begin{array}{l}\text { Argissolo Vermelho } \\
\text { Distrófico }\end{array}$ \\
\hline PB7 & $\begin{array}{l}\text { Pasto queimado, entre pasto e } \\
\text { rodovia na zona rural }\end{array}$ & $\begin{array}{c}455995 \\
7520956\end{array}$ & $\begin{array}{l}8-20 \% \\
\text { Ondulado }\end{array}$ & $\begin{array}{l}\text { Argissolo Vermelho } \\
\text { Distrófico }\end{array}$ \\
\hline
\end{tabular}

* Para fins acadêmicos e com base em parâmetros limitantes da capacidade de suporte, pastagem considerada não degradada, de acordo com os níveis de degradação propostos por Dias Filho (2011).

Para avaliação da qualidade do solo nessas áreas foi utilizada a metodologia RAQS, proposta por Souza et al. (2016), por meio de indicadores visuais semi-quantitativos dos aspectos físicos e biológicos do solo. Os indicadores utilizados foram: cor e odor do solo; suscetibilidade à erosão; pedregosidade; estrutura e consistência do solo; compactação; porosidade; profundidade da camada superficial e quantidade de serapilheira; atividade de oxigenase no solo; presença de fauna do solo como minhocas e outros invertebrados; cobertura vegetal; diversidade vegetal; porte e estratificação da vegetação; infestação de plantas daninhas e presença ou rastros de animais de maior porte como anfíbios, répteis, aves e mamíferos.

Cada área foi dividida em quatro subáreas, sendo avaliadas visualmente por quatro avaliadores previamente treinados, os quais atribuíram as notas: 0 (pobre); 1 (moderado); 2 (bom) e 3 (ótimo), de forma independente, para cada atributo da 1778

Souza; K. R., Melloni; R., Ferreira, G. M. R. 
metodologia. O peso atribuído a cada atributo variou de 1 a 3, conforme Souza et al. (2016).

O IQS foi calculado pela equação $1 \mathrm{com}$ expressão dos resultados em porcentagem $(\%)$ para facilitar a comparação entre as diferentes áreas.

$$
I Q S=\frac{\left(\sum N \times P\right) \times 100}{n}
$$

$\mathrm{N}$ : nota atribuída a cada atributo nas subáreas

P: peso de cada atributo

n: número de avaliadores por subárea

Com as médias de cada atributo visual determinado pelos avaliadores foi gerado um perfil de similaridade entre as áreas, por meio do método "Complete linkage" e matriz de distâncias euclidianas. No entanto, para verificar a relação entre as áreas e os atributos visuais analisados aplicou-se análise multivariada de componentes principais, também por meio do software Statistica 7.0 (Statsoft, 2004).

\section{Resultados e discussão}

Os IQS calculados para as áreas de pastagem estão apresentados na figura 1, bem como o resultado da análise de agrupamento com base em todos os atributos visuais avaliados. $\mathrm{O}$ dendrograma revelou alta similaridade entre as áreas, sendo somente três grupos formados a $70 \%$ de similaridade, sendo um exclusivo com a área CAP2 e outros com 4 e 9 grupos.

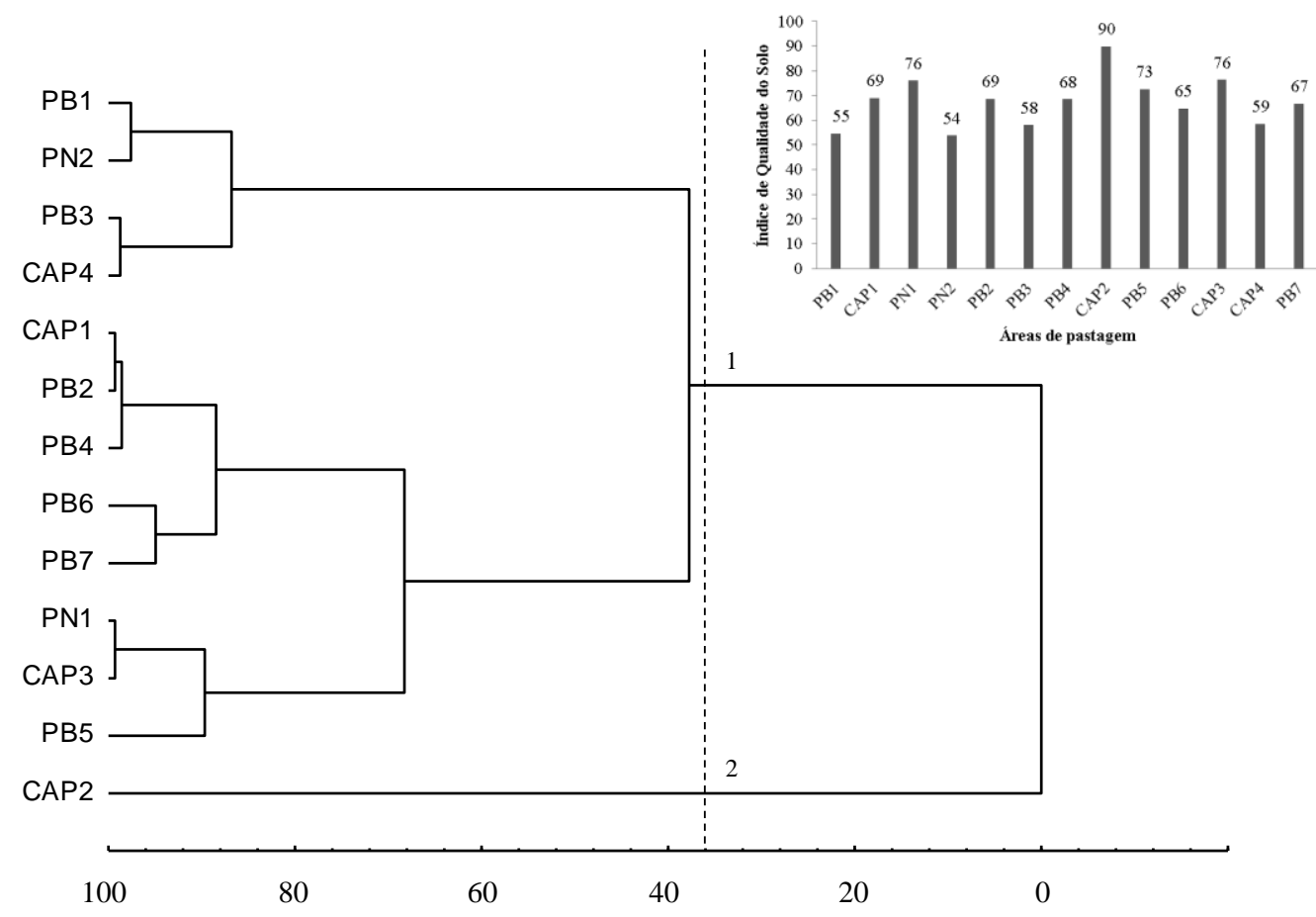

Figura 1. Dendrograma de similaridade das áreas de pastagem construído exclusivamente por meio de seus índices de qualidade do solo (colorido). PB1 (Pasto visualmente bom 1), CAP1 (Capoeira, pasto em estágio intermediário de sucessão 1), PN1 (Pasto com início de degradação), PN2 (Pasto degradado), PB2 (Pasto visualmente bom 2), PB3 (Pasto visualmente bom 3), PB4 (Pasto visualmente bom 4), CAP2 (Capoeira, pasto em estágio intermediário de sucessão 2), PB5 (Pasto visualmente bom 5), PB6 (Pasto visualmente bom 6), CAP3 (Capoeira, pasto em estágio intermediário de sucessão 3), CAP4 (Capoeira, pasto em estágio intermediário de sucessão 4), PB7 (Pasto queimado). 
Dessa forma, pode-se verificar que, pelo fato do IQS envolver atributos visuais relacionados a solo, planta e animais, nem sempre um pasto considerado visualmente bom somente pelos seus atributos homogeneidade de cobertura e baixa erodibilidade (PB1, PB2, PB3, PB4, PB6) apresenta maiores valores de IQS. Nesse sentido, verifica-se que valores de IQS acima de 70\% também foram obtidas em áreas em início de degradação ou em estágio intermediário de sucessão, enquanto notas abaixo de $60 \%$ também foram observadas em áreas com pasto visualmente bom, como a PB1 e PB3.

Alguns atributos visuais relacionados à vegetação, como índice de diversidade, porte e estratificação e sucessão na vegetação, são considerados para o cálculo na metodologia RAQs e, não necessariamente, estão relacionados a boas pastagens (Souza et al., 2016). Normalmente, áreas consideradas com pastos bons tendem a se apresentar baixo nível ou ausência de degradação, boa porcentagem de cobertura vegetal, fornecendo forragem em quantidade suficiente para alimentação animal (Dias Filho, 2011), mas com baixa diversidade vegetal e ausência de sucessão. Assim, para casos de avaliação de qualidade de pastagens visando produção animal, deve-se ter cautela ao se usar um IQS, já que baixos valores, resultados de baixas notas nesses dois atributos, podem causar equívocos de interpretação. Nesse sentido, para esses pastos, onde os processos de sucessão não ocorrem ou são controlados por manejo, os atributos relacionados à diversidade vegetal e sucessão não devem ser considerados para o cálculo do IQS.

Área de pastagem que sofreu ação de queimada, mas que esteja em processo de recuperação, como a PB7, pode apresentar valores intermediários de IQS e se agrupar com áreas consideradas boas (Figura 1). Isso ocorreu, em virtude, principalmente, do cálculo do IQS envolver atributos relacionados à sucessão ecológica e à diversidade de plantas pioneiras, comuns nesses casos de recuperação que não envolveu a presença de animais (Scasta et al., 2016).
O impacto do fogo no solo está relacionado com o grau de severidade das queimadas (Shaw et al., 2016). Nessas áreas, indicadores visuais podem ser úteis em determinar o grau de degradação dos solos, principalmente para tomada de decisão logo após a queimada, uma vez que a remoção da camada vegetal, aliada à presença de chuva e tipo de relevo do terreno, podem intensificar processos de erosão (Vega et al., 2013). No presente trabalho, a técnica de avaliação visual do IQS, proposta por Souza et al. (2016), possibilitou o agrupamento dessa área com outras de maiores notas (Figura 1) em função das altas notas obtidas para atributos relacionados à vegetação, como sucessão na vegetação, presença de plantas daninhas, composição e diversidade na vegetação. Portanto, áreas fortemente impactadas, mas em intenso processo de recuperação, podem apresentar valores variáveis de IQS, os quais podem ser utilizados para o monitoramento da recuperação de sua qualidade ao longo do tempo.

Outro fator que pode interferir na variação do IQS é a topografia onde a área está inserida. As áreas de pastagem estudadas apresentaram topografia diferenciada (Tabela 1), principalmente relacionado ao relevo do terreno, variando-se entre terrenos planos a fortemente ondulados. Entretanto, mesmo a topografia do local podendo influenciar na qualidade do solo, principalmente por ação das chuvas (Lozano-Garcia et al., 2016), os maiores IQS (áreas CAP2, PN1, CAP3 e PB5) não se relacionaram à topografia, apresentando-se em relevos suave-ondulado (CAP3), ondulado (PN1) até forte-ondulado (CAP2 e PB5). Dessa forma, os valores de IQS variaram em função dos atributos de solo, vegetação e fauna (Souza et al., 2016), mas não da topografia do relevo onde as áreas estão.

Para verificar quais atributos visuais apresentaram maior relação com os resultados do agrupamento das áreas (Figura 1), foi empregada análise multivariada de componentes principais (CP). Os CP 1 e 2 e 3 acumulados explicaram $71,94 \%$ da variância total dos dados e seus autovetores estão apresentados na tabela 2 . 
Revista Brasileira de Geografia Física v.11, n.05 (2018) 1776-1785.

Tabela 2. Autovetores extraídos dos componentes principais

\begin{tabular}{cccc}
\hline Variables & CP 1 & CP 2 & CP 3 \\
\hline Cor e odor do solo (C.O.) & 0,06977 & 0,46632 & $-1,15228$ \\
Susceptibilidade à erosão (Eros.) & $\underline{-2,04659}$ & $\underline{2,20926}$ & $-0,64683$ \\
Pedregosidade (St.) & $\underline{-3,20255}$ & $\underline{-2,57508}$ & 1,68231 \\
Estrutura e consistência do solo (Strut.) & $\underline{-2,14293}$ & $-1,02566$ & $-1,47061$ \\
Compactação (Comp.) & 0,21759 & 0,07677 & $-1,22865$ \\
Porosidade (Por.) & $-0,88252$ & $\underline{-1,37050}$ & $-0,62932$ \\
Profundidade da camada superficial (Prof.) & $\underline{-1,46153}$ & $-0,85404$ & $-0,96330$ \\
Quantidade, decomposição e incorporação da & $\underline{2,43063}$ & $\underline{2,01356}$ & 0,62269 \\
Presença e atividade de organismos (Ox.) & $\underline{2,62243}$ & 1,25902 & $-0,64516$ \\
Fauna do solo (Inv.) & 1,08575 & $\underline{2,10991}$ & $\underline{2,02997}$ \\
Índice da cobertura (Cob. Veg.) & $\underline{-3,36158}$ & 0,95655 & 0,56887 \\
Índice de diversidade (Div. Veg.) & $\underline{1,69848}$ & $-0,15305$ & $\underline{2,78852}$ \\
Porte e estratificação (Port.) & $-0,52887$ & $\underline{-1,36913}$ & $\underline{2,02889}$ \\
Vigor da Vegetação (V. Veg.) & $\underline{-1,76623}$ & $\underline{1,75779}$ & $-1,00920$ \\
Sucessão na vegetação (Suc. Veg.) & $\underline{4,23604}$ & $\underline{-1,40713}$ & $-0,89426$ \\
Infestação de plantas daninhas (Dan.) & $\underline{3,29391}$ & $\underline{-2,15108}$ & $-0,77543$ \\
Fauna (anfíbios, répteis, aves e mamíferos) (Vert.) & $-0,26179$ & 0,05651 & $-0,30620$ \\
\hline Subling
\end{tabular}

Sublinhados os valores superiores a |1.30000| apenas para fins de interpretação.

No presente estudo, a maioria dos atributos relacionados ao solo apresentou maiores relações aos CP1 e CP2, sendo pedregosidade, presença e atividade de organismos, serapilheira, estrutura e consistência, susceptibilidade à erosão e fauna do solo os mais fortemente representativos e indicados para outros estudos de avaliação da qualidade de solo de pastagem. Em relação aos atributos de vegetação, a maioria também esteve mais relacionada aos CP1 e CP2, sendo índice de cobertura, sucessão na vegetação, índice de diversidade, porte e estratificação, e infestação de plantas daninhas os mais fortemente relacionados e aqueles mais indicados para avaliação da qualidade visual de pastagem. Para os atributos fauna silvestre (vertebrados), cor e odor do solo, e compactação não se verificou efeito significativo e discriminante para avalição da qualidade das áreas de pastagem em estudo, não sendo, portanto, bons indicadores para esse estudo. Tal resultado não contraria a importância desses atributos para a qualidade do solo (Galli et al., 2015; Souza et al., 2016; Velásquez et al., 2007), mas indica a dificuldade de obtenção desses dados por métodos visuais e padronização dos índices de qualidade do solo em condições de campo (Obade e Lal, 2016).
Áreas de pastagem em diferentes graus de degradação, em processo natural de recuperação, são importantes locais para se estudar a dinâmica dos processos biológicos, bem como a importância de diversos organismos para o ambiente. Avaliando áreas em recuperação, Paula et al. (2006) demonstraram função essencial de invertebrados, como as minhocas, nos processos de sucessão e restauração do solo, devido, principalmente, à disponibilização de nitrogênio para as plantas. Naquele estudo, áreas que apresentaram maior diversidade e abundância desse grupo apresentaram processo mais rápido de recuperação. Modificações nas qualidades física e química do solo podem influenciar diretamente a presença de invertebrados, os quais apresentam significativa importância aos processos do solo pois apresentam capacidade de alterar as características físicas e químicas, bem como seus processos microbiológicos (Kim et al., 2015).

Em virtude das relações entre as áreas e atributos serem maiores com os componentes principais CP1 e CP2 (Tabela 2), somente esses serão apresentados (Figura 2). Houve a formação de três grupos, sendo um grupo I formado pelas áreas de pasto considerado visualmente bom (PB1, PB2,

Souza; K. R., Melloni; R., Ferreira, G. M. R. 
PB4, PB5 e PB6), no quadrante superior esquerdo, com fortes relações entre qualidade do solo e qualidade da vegetação, como maior porcentagem de cobertura e vigor da vegetação, baixa presença de plantas daninhas, baixa sucessão ecológica e diversidade vegetal, além de bons aspectos de solo como baixa susceptibilidade à erosão e presença de macrofauna e seus vestígios. Pelos resultados desse grupo I sugere-se que um pasto considerado visualmente de boa qualidade para criação de animais deva apresentar esse conjunto de indicadores, e não somente considerando os valores de IQS observados anteriormente (Figura 2). De fato, baixos valores de IQS para pastos visualmente propícios à criação animal (como PB1) sugerem que a interpretação de alguns atributos visuais pode causar interpretações controversas, já que nem sempre há relação direta entre sustentabilidade ambiental (com maior diversidade vegetal, sucessão vegetal, presença de plantas daninhas) e ambiente propício para criação animal.

Outro grande grupo II foi formado pelas demais áreas de capoeira e degradadas (CAP1, CAP3, CAP4, PN1, PN2), com relações positivas entre qualidade do solo e seus atributos como profundidade da camada superficial, estrutura e consistência, porosidade, pedregosidade, porte e estratificação da vegetação, e negativas com a presença de invertebrados, serapilheira, atividade dos organismos do solo, compactação, coloração e odor do solo.

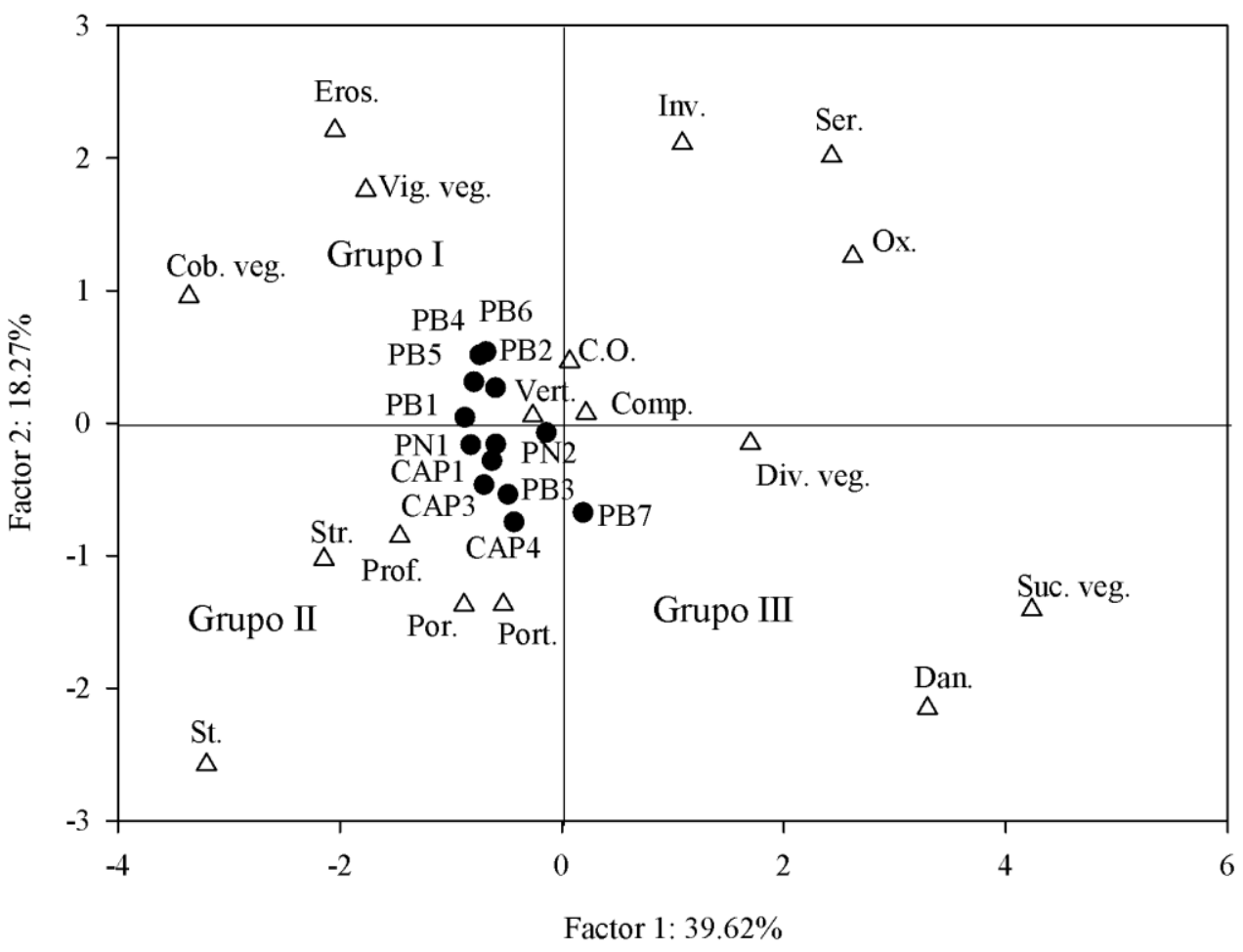

Figura 2. Gráfico de componentes principais. PB1 (Pasto visualmente bom 1), CAP1 (Capoeira, pasto em estágio intermediário de sucessão 1), PN1 (Pasto com início de degradação), PN2 (Pasto degradado), PB2 (Pasto visualmente bom 2), PB3 (Pasto visualmente bom 3), PB4 (Pasto visualmente bom 4), CAP2 (Capoeira, pasto em estágio intermediário de sucessão 2), PB5 (Pasto visualmente bom 5), PB6 (Pasto visualmente bom 6), CAP3 (Capoeira, pasto em estágio intermediário de sucessão 3), CAP4 (Capoeira, pasto em estágio intermediário de sucessão 4), PB7 (Pasto queimado). Cor e odor do solo (C.O); Susceptibilidade à erosão (Eros.); Pedregosidade (St.); Estrutura e consistência do solo (Str.); Compactação (Comp.); Porosidade (Por.); Profundidade da camada superficial (Prof.); Quantidade, decomposição e incorporação da serapilheira (Serr.); Presença e atividade de organismos (Ox.); Fauna do solo (Inv.); Índice da cobertura (Cob. Veg.); Índice de diversidade (Div. Veg.); 
Porte e estratificação (Port.); Vigor da Vegetação (V. Veg.); Sucessão na vegetação (Suc. Veg.); Infestação de plantas daninhas (Dan.); Fauna (anfíbios, répteis, aves e mamíferos) (Vert.).

A área PB7 foi exclusiva do grupo III como resultado da queimada, a qual reduziu a cobertura vegetal e o vigor das plantas, com consequente aumento da suscetibilidade à erosão, apesar dos processos de sucessão estarem ocorrendo sem interferência animal. O tempo de recuperação dessa área ainda não foi suficiente para que a sua qualidade visual melhorasse e evoluísse para aquelas em estágio maior de sucessão (grupo II), apresentando-se, na época de avaliação, com qualidade inferior e oposta à observada nos pastos considerados de boa qualidade (grupo I).

Dessa forma, se verifica a extrema sensibilidade desses atributos visuais em discriminar as diferentes áreas de pastagem, possibilitando o seu agrupamento em função do solo, plantas e animais, auxiliando a tomada de decisão para implantação de medidas de recuperação, e relacionando a qualidade do solo com o uso da terra (Obade e Lal, 2016; Lima et al., 2013.). A avaliação visual da qualidade de pastagens sob diferentes tipos de manejo, além de não ter custo adicional e ampliar o vínculo homemambiente, facilita o acompanhamento ao longo do tempo e pode trazer benefícios, não somente ao produtor, mas ao ambiente onde estão inseridas (Chaves et al., 2017).

\section{Conclusões}

A avaliação de áreas de pastagem, por meio de atributos relacionados a solo, plantas e fauna, possibilitou, com baixo custo e eficiência, acesso a sua qualidade ambiental.

A qualidade visual das áreas de pastagem variou e esteve mais fortemente relacionada com a vegetação para pastos considerados visualmente bons, com atributos de solo para pastos em diversos níveis de degradação e em recuperação, e com o impacto da queimada.

Os atributos fauna silvestre (vertebrados), cor e odor do solo e compactação não foram considerados bons indicadores da qualidade dos solos das áreas de pastagem estudadas.

\section{Agradecimentos}

À Coordenação de Aperfeiçoamento de Pessoal de Nível Superior (Capes) e à Fundação de
Amparo à Pesquisa do Estado de Minas Gerais (Fapemig), pelo apoio.

\section{Referências}

Askari, M. S., Holden, M. M., 2015. Quantitative soil quality indexing of temperate arable management systems. Soil and Tillage Research 150:57-67.

Bunemann, E. K., Bongiorno, G., Bai, Z., Creamer, R. E., Deyn, G. D., Goede, R., Fleskens, L., Geissen, V., Kuyper, T. W., Mader, P., Pulleman, M., Sukkel, W., Groenigen, J. W. V., Brussard, L., 2018. Soil quality - A critical review. Soil Biology and Biochemistry 120:105125.

Cavagnaro, T. R., Cunningham, S. C., Fitzpatrick, S., 2016. Pastures to woodlands: changes in soil microbial communities and carbon following reforestation. Applied Soil Ecology 107: 24-32.

Chaves, H. M. L., Lozada, C. M. C., Gaspar, R. O., 2017. Soil quality index of and oxisol under different land uses in the Brazilian savannah. Geoderma Regional 10:183-190.

Cherubin, M. R., Franci, A. L. C., Cerri, C. E. P. Oliveira, D. M. S., Davies, C. A., Cerri, C. C., 2015. Sugarcane expansion in Brazilian tropical soil - Effects of land use change on soil chemical attributes. Agriculture, Ecosystems \& environment 211: 173-184.

Crittenden, S. J., Poot, N., Hienen, M., Balen, D. J. M. V., Pulleman, M. M., 2015. Soil physical quality in contrasting tillage system in organic and conventional farming. Soil Tillage Research 154: $136-144$.

Dias-Filho, M. B., 2011 Degradação de pastagens: processos, causas e estratégias de recuperação. 4. ed. Embrapa Amazônia Oriental, Belém, PA.

Fusaro, S., Gavinelli, F., Lazzarini, F., Paoletti, M. G., 2018. Soil biological quality index based on 
earthworms (QBS-e). A new way to use earthworms as bioindicators in agroecosystems. Ecological Indicators 93:1276-1292.

Galli, L., Bonacchi, A., Capurro, M., Conti, I., Crovetto, F., Ferrari, C., Conti, F. D., Menta, C., 2015. Assessment of the impact of trampling on soil Arthropoda in a Mediterranean habitat. Acta Societatis Zoologicae Bohemicae 79: 193-198.

Guimarães, R. M. L., Ball, B. C., Tormena, C. A., Giarola, N. F. B., Silva, A. P., 2013. Relating visual evaluation of soil structure to other physical properties in soils of contrasting texture and management. Soil Tillage Research 127: 9299.

Kim, Y. N., Robinson, B., Boyer, S., Zhong, H. T., Dickinson, N., 2015. Interactions of native and introduced earthworms with soils and plant rhizosphere in production landscapes of New Zealand. Applied Soil Ecology 96: 141-150.

Kosmas, C., Karamesouti, M., Kounalaki, K., Detsi, V., Vassiliou, P., Salvatti, L., 2016. Land degradation and long-term changes in agropastoral system: An empirical analysis of ecological resilience in Asteroussia - Crete (Greece). Catena 147: 194-204.

Lima, A. C. R., Brussard, L., Totola, M. R., Hoogmoed, W. B., Goede, R. G. M., 2013. A functional evaluation of three indicators sets for assessing soil quality. Applied Soil Ecology 64:194-200.

Lozano-Garcia, B., Parras-Alcantara, L., Brevick, E. C., 2016. Impact of topographic aspect and vegetation (native and reforested areas) on soil organic carbon and nitrogen budgets in Mediterranean natural areas. Science of the Total Environment 544: 953-970.

Moncada, M. P., Gabriel, D., Cornelis, W. M., 2014. Data-driven analysis of soil quality indicators using limited data. Geoderma 235: 271-278.

Mudge, P., Kelliher, F. M., Knight, T. L., O’Connel, D., Fraser, S., Schipper, L. A., 2016. Irrigating grazed pasture decreases soil carbon and nitrogen stocks. Global Change Biology 1: 1-10.
Naderi-Boldaji, M., Keller, T., 2016. Degree of soil compactness is highly correlated with soil physical quality index S. Soil and Tillage Research 159:41-46.

Nicholls, C. I., Altieri, M. A., Dezanet, A., Lana, M., Feistauer, D., Ouriques, M., 2004. A rapid, farmer-friendly agroecological method to estimate soil quality and crop health in vineyard system. Biodynamics 1: 33-40.

Obade, V. P., Lal, R., 2016. A standardized soil quality index for diverse field conditions. Science of the total environment 541: 424-434.

Obade, V. P., Lal, R., 2016. Towards a standard technique for soil quality assessment. Geoderma 265:96-102.

Oliveira, G. G., Oliveira, G. C., Silva, E. A., Carducci, C. E., Barbosa, S. M., Silva, B. M., 2015. Indicadores de qualidade física para Argissolos sob pastagens nas regiões leste e sul de Minas Gerais. Amazonian Journal of Agricultural and Environment Sciences 58:388395.

Paula, R. C., Silveira, R. M. L., Rocha, M. M., Izzo, T. J., 2016. The restoration of termite diversity in different reforested forests. Agroforest System 90: 395-404.

Paz-Kagan, T., Ohana-Levi, M., Herrmann, I., Zaadu, E., Henkin, Z., Karnieli, A., 2016. Grazing intensity effects on soil quality: A spatial analysis of a Mediterranean grassland. Catena 146: 100-110.

Pulido, M., Schnabel, S., Contador, J. F. L., Parra, J. L., Gonzalez, F., 2016. The impact of heavy grazing on soil quality and pasture production in rangelands in SW Spain. Land Degradation and Development 1: 1-12.

Raiesi, F., Kabiri, V., 2016. Identification of soil quality indicators for assessing the effect of different tillage practices through a soil quality index in a semi-arid environment. Ecological Indicators 71:198-207.

Scasta, J. D., Duchardt, C., Engle, D. M., Miller, J. R. Debinski, D. M., Harr, R. N., 2016. 
Constraints to restoring fire and grazing ecological processes to optimize grassland vegetation structural diversity. Ecology Engineering 95: 865-875.

Shaw, E. A., Denef, K., Tomasel, C. M., Cotrufo, M. F., Wall, D. H., 2016. Fire effects root decomposition, soil food web structure, and carbon flow in tall grass prairie. Soil 2: 199-210.

Souza, K. R., Melloni, R., Ferreira, G. M. R., Souza, S. M. P., Rezende, J. T., 2016. Proposta da metodologia RAQS para avaliação visual da qualidade do solo. Revista Brasileira de
Geografia Fisica 9: 1815-1824.

STATSOFT, Inc., 2004. Statistica (Data Analysis Software System), version 7.

Vega, J. A., Fonturbel, T., Merino, A., Fernandez, C., Ferreiro, A., Jimenez, E., 2013. Testing the ability of visual indicators of soil burn severity to reflect changes in soil chemical and microbial properties in pine Forest and shrubland. Plant Soil 369: 73-91. 